\title{
Evaluar para el aprendizaje. Evaluación de la comprensión de documentos no escritos
}

\author{
Jesús Alonso-Tapia ${ }^{1}$, Helena Garrido-Hernansaiz ${ }^{1}$
}

${ }^{1}$ Departamento de Psicología Biológica y de la Salud, Universidad Autónoma de Madrid, Madrid.

\section{España}

Correspondencia: Jesús Alonso-Tapia. Departamento de Psicología Biológica y de la Salud, Universidad Autónoma de Madrid, calle Ivan Pavlov, 6. 28049, Madrid. España. E-mail: jesus.alonso@uam.es

(C) Education \& Psychology I+D+i and Ilustre Colegio Oficial de Psicología de Andalucía Oriental (Spain) 


\section{Resumen}

Introducción. Pese a que la comprensión de documentos no escritos (DNE) forma parte del currículo oficial español de Educación Secundaria, no existe investigación respecto a cómo se enseña y evalúa. El principal objetivo de este estudio es analizar cómo los profesores consideran que debe evaluarse esta capacidad para ayudar a decidir qué apoyos dar al alumnado.

Método. Se desarrolló en primer lugar un modelo de lo que implica la competencia de comprensión de DNE. A continuación, 148 profesores de Educación Primaria en España diseñaron tareas de evaluación de dos DNE con el fin señalado. Posteriormente, dos jueces evaluaron las tareas propuestas de acuerdo al modelo inicialmente especificado, y se analizó el grado en que la evaluación de los profesores se ajusta al modelo ideal propuesto mediante análisis porcentuales por categorías y un análisis loglineal jerárquico.

Resultados. Las evaluaciones de los profesores fueron poco sistemáticas y se ciñeron a tareas de un nivel de complejidad generalmente muy bajo, incluyendo además casi siempre preguntas ambiguas e irrelevantes para identificar la comprensión. Las tareas propuestas por los profesores respondieron a perfiles diferentes, alguno de los cuales se ajusta al modelo ideal propuesto.

Conclusión. La formación del profesorado debería introducir cambios curriculares para favorecer la adquisición de la competencia de comprender DNE, especialmente para poder detectar las dificultades de los alumnos y ayudarles a superarlas.

Palabras Clave: Evaluación para el aprendizaje, documentos no escritos, comprensión, formación del profesorado. 


\begin{abstract}
Introduction. Despite the fact that the capacity of non-written sources (NWS) understanding is included in the Spanish secondary education curriculum, no research has addressed this competence. The main objective of this study is analysing how teachers consider NWS understanding that should be assessed to help decide which kind of support give to students.

Method. First, a model was developed of what NWS understanding implies. Then, 148 primary school teachers in Spain designed tasks to assess the understanding of two NWS. Following this, two judges assessed the proposed tasks according to the initially specified model. We analyzed the fit of teachers' assessment tasks to the proposed model by conducting percentage analyses and a hierarchical loglinear analysis.
\end{abstract}

Results. Teachers' assessments were shown to be unsystematic and were generally composed of tasks of a low level of complexity. Nearly all of them included ambiguous questions or questions which were irrelevant for testing understanding. The proposed tasks corresponded to different profiles, some of which fitted the proposed ideal model.

Conclusion. Some changes should be made in teacher education in order to foster students' competency for NWS understanding, especially to detect students' difficulties so that they can be presented with suitable feedback.

Keywords: Assessment for learning, non-written sources, understanding, teacher education. 


\section{Introducción}

La evaluación es uno de los factores que más influye en la motivación por aprender (Alonso-Tapia y Fernández, 2008; Alonso-Tapia y Pardo, 2006) y, a través de ésta, en el aprendizaje (Alonso-Tapia, 1997, 2001; Segers, Dochy y Cascallar, 2003; Mingorance Arnáiz y Calvo Bernardino, 2013). Por ello, los profesores deben conocer qué procedimientos de evaluación favorecen más la motivación, ya que la evaluación afecta al interés y esfuerzo por aprender del alumno.

Dochy (2005) distingue dos perspectivas en torno a la evaluación: la cultura del examen, que busca identificar la cantidad de conocimiento 'depositado' en la mente del estudiante (assessment 'of' learning), y la cultura de la evaluación, que intenta proporcionar información al profesor y al alumno para superar las dificultades y autorregular el aprendizaje (assessment 'for' learning). El profesor, dependiendo de la perspectiva, crea contextos de evaluación diferentes que, a su vez, pueden afectar al esfuerzo del alumno. Es importante también saber qué indicadores de comprensión son válidos ya que, aun trabajando desde la cultura de la evaluación, pueden considerarse válidos indicadores que no lo son y ofrecer al alumno retroalimentación de baja calidad o inadecuada.

Además, los profesores no cambian sus métodos habituales de evaluación salvo que sean conscientes de la discrepancia existente entre la información que proporcionan los métodos que utilizan y el tipo de evidencia que informaría de hecho sobre las competencias a adquirir y que permite saber cómo ayudar a los alumnos para que puedan mejorarlas (PérezLandazábal y Moreno, 1998). Por esta razón es necesario ayudar a los profesores a tomar conciencia de tal discrepancia, y para ello es imprescindible conocer las características de las evaluaciones que realizan.

Habitualmente, los métodos de evaluación utilizados son deficientes (Black y Wiliam, 1998, Segers et al., 2003). Pérez de Landazábal, Varela y Alonso-Tapia (2012) encontraron, en ciencias experimentales, que los profesores utilizaban mayoritariamente tareas de baja complejidad al evaluar la comprensión de conceptos, de textos, de tablas y gráficos y el pensamiento científico. El mismo resultado ha sido encontrado en ciencias sociales para la comprensión de la causalidad histórica (Alonso-Tapia, Asensio, López, Carriedo y Rychecki, 2007) y de tablas y gráficos (Alonso-Tapia y Hernansaiz-Garrido, 2013), donde también se 
encontró una tendencia a realizar preguntas sobre conocimiento de hechos. Estos modos de evaluación no permiten proporcionar a los alumnos las ayudas adecuadas para superar las dificultades de comprensión.

El presente estudio analiza si los profesores conocen qué tareas son adecuadas para evaluar la comprensión de documentos no escritos (DNE) en el contexto de las Ciencias Sociales. Se consideran DNE las producciones de carácter no verbal elaboradas por una sociedad con o sin intencionalidad comunicativa. Son utilizados habitualmente en diferentes ámbitos en de las ciencias naturales y sociales para obtener información sobre la sociedad que los ha generado, por ejemplo en el estudio de la historia de la geología (Oldroyd, 1999) o de los sistemas de salud públicos en África (Waite, 1987). Incluyen objetos de distinta naturaleza tales como herramientas, utensilios, armas, edificios, esculturas, pinturas, fotografías, medios de transporte, vestidos, calzado, etc. Comprender los DNE consiste en extraer la información que encierran sobre la sociedad que los creó, de modo similar a como hacen los arqueólogos y paleontólogos.

Dado el valor informativo de los DNE, el currículo español de Ciencias Sociales establece como objetivo que los alumnos desarrollen la competencia de interpretar su significado. Los libros de texto y los profesores, en consecuencia, los utilizan como ilustraciones cuando describen las sociedades y explican las causas de los cambios históricos. Sin embargo, hasta donde los autores conocen, tras revisar la abundante bibliografía sobre evaluación de competencias, aunque existen estudios sobre cómo los profesores evalúan de hecho en Ciencias Sociales (Villa y Alonso-Tapia, 1996), no existen estudios sobre la comprensión de DNE ni sobre la evaluación de tal comprensión entre los 12 y los 18 años. Esto puede deberse parcialmente a la ausencia de una comprensión adecuada de lo que implica esta competencia y, en consecuencia, de un modelo teórico. Por ello, este trabajo busca presentar un modelo teórico sobre la comprensión de DNE que sirva, además, de guía didáctica y de evaluación de esta competencia. Posteriormente, el trabajo busca también valorar si la forma de evaluación de los profesores se ajusta al modelo presentado. Obviamente, otros modelos pueden ser posibles; nuestra intención es establecer un punto de partida desde el que comenzar a abordar el estudio del problema planteado. 
Supuestos desde los que Evaluar la Comprensión de DNE

\section{Definición del problema}

La información utilizada para representar la estructura y el dinamismo social en un momento dado y el cambio de las sociedades a lo largo del tiempo se encuentra no sólo en documentos escritos -textos, tablas y gráficos-, sino también en distintas fuentes no escritas: esculturas, pinturas, grabados, construcciones civiles, militares o religiosas, objetos diversos... Ahora bien, ¿qué implica la capacidad de analizarlos e interpretar su significado? ¿De qué depende que los alumnos comprendan o no el valor de la información que contienen? ¿Y cómo evaluar no sólo si los alumnos comprenden su significado, sino también a qué se deben sus fallos de comprensión, para poder ayudarles?

\section{¿Qué implica comprender el significado histórico-social de un DNE?}

Conocer las dimensiones que enmarcan el significado del objeto. Aunque no hay estudios sobre cómo novatos y expertos comprenden los DNE (de forma probablemente distinta, dada la diferencia de conocimientos entre unos y otros), es posible que el experto tienda a ubicar espontáneamente el documento dentro del contexto histórico-social en que se originó y a valorarlo desde su modelo conceptual sobre dicho contexto. Por ejemplo, un experto probablemente piense que comprender el significado del Pórtico de la Gloria implica saber que es un pórtico románico de función religiosa y didáctica. En consecuencia, a la hora de evaluar qué valor documental atribuyen los alumnos a este documento histórico, es posible que les pregunte por su función y por el estilo artístico al que pertenece.

Esto, sin embargo, implicaría centrarse en el principal significado ya atribuido al documento, analizándolo como obra de arte. Este análisis no supone analizar el documento como fuente, sino como ejemplo ilustrativo de un modelo conceptual ya construido sobre un estilo artístico. Además, no evalúa si el alumno ha desarrollado un esquema de cuestiones válido para el análisis de los DNE, esquema que a menudo sigue el experto de forma casi inconsciente al encontrarse con un documento desconocido. En la Figura 1 se presenta un posible esquema válido que se comenta a continuación. 


\section{Guion para el análisis de Documentos No Escritos}

a) Descripción y función: ¿Qué es? ¿Qué características tiene? ¿Para qué puede servir?

b) Lbicación espacio-temporal: ¿Dónde y cuándo aparece?

c) Implicaciones básicas:

1. ¿Qué fiuentes de recursos matcriales se requicren para su zlaboración?

2. ¿Qué implica la mayor o menor accesibilidad a las mismas en el lugar de su ubicación?

3. ¿Qué grado de elaboración implica el mąerial de que está hecho?

4. ¿Qué lipo de conocimientos tecnológicos se requieren paza su elaboración?

5. ¿Qué can-idad de personas se requeririan para la consecución y elaboración de los mismos?

6. ¿Qué tipo y grado de orgarización social y politica se recucririan para su elaboración

7. ¿Qué tipo de recursos económicos se requieren para st: elaboración?

8. ¿Se requiere alguna condición especial para que puedan existir tales recursos económincos?

9. ¿Qué cantidad de tiempo se requiere para la realización del objeto en cuestión? ${ }_{b} \mathrm{Y}$ para su difusión?

10. ¿Qué valores, creencias, metas, sensibilidad y personalidad parece reflejar la existencia, naturaleza y (eventualmente) el contenido de la fuente u objezo analizado?

11. ¿Qué impacto ha podido tener la calegoria de objetos a la que pertenece en el cambio social?

12. ¿A qué se debe su sittación y estado accual?

Figura 1. Guion para el análisis de DNE.

Descripción de la fuente: características, naturaleza y función. Identificar las características, naturaleza y función probable de la fuente es lo primero que los alumnos deben poder hacer antes de extraer la información que contiene (Trepat i Carbonell, 1995). Sin embargo, para esto es necesario conocer previamente las características de la categoría de objetos a que pertenece. Por ejemplo, aunque un alumno pueda describir un trillo, sin conocer qué función tienen los trillos es muy difícil que pueda identificarlo como instrumento agrícola y extraer posteriormente las implicaciones derivadas. Por ello, lo primero a evaluar es si conocen de qué objeto se trata. Aunque en muchos casos no sea necesario por tratarse de objetos conoci- 
dos, si no lo saben será preciso ayudarles a descubrir su posible naturaleza a partir del análisis de sus características o, dependiendo del caso, decirles directamente qué es.

Ubicación espacio-temporal. Los objetos cobran significado en función del contexto y momento en que son creados, desarrollados y utilizados. El significado del objeto en relación con su contexto sociocultural varía dependiendo de dicho contexto; por ejemplo, no tienen el mismo significado las ruinas de una capilla cristiana excavadas en Roma que en la India. Además, es preciso diferenciar entre preguntarse dónde y cuándo aparece el objeto y responderse. Lo primero forma parte de la serie de cuestiones a plantearse para valorar un DNE, un conocimiento procedimental que es necesario enseñar de modo explícito. Lo segundo depende de los conocimientos factuales o conceptuales previos que se tienen sobre el objeto en cuestión, algo que requiere una ayuda diferente. En consecuencia, será preciso evaluar tanto si los alumnos se plantean la pregunta (o la tienen en cuenta para buscar el significado del objeto) como si son capaces de responder en función de sus conocimientos previos.

Análisis de implicaciones. Una vez descrito, identificado y ubicado el objeto (en la medida de lo posible), es el momento de extraer información del mismo que pueda ayudar a reconstruir su contexto de aparición. ¿Qué preguntas debería hacerse el alumno para ello? Debería ser capaz de plantearse espontáneamente las siguientes preguntas y examinar las implicaciones derivadas de ellas:

1. ¿Qué recursos materiales se requieren para su elaboración? Todo objeto está hecho de algún material. Considérese la pintura de Altamira (pigmentos), el Partenón (piedra) o una moneda (metal). ¿Por qué es importante considerar los materiales? Porque necesitan estar disponibles, pueden requerir distinto grado de transformación y elaboración para poder ser utilizados y pueden requerir mayor o menor fuerza de trabajo para conseguirlos, transformarlos y utilizarlos.

2. ¿Qué implica la mayor o menor accesibilidad de los materiales? Una vez identificado el material, es preciso aprender a preguntarse si es fácilmente asequible. Si no está disponible cerca del objeto, requiere transportarlo, lo que debe llevar a plantearse dos cuestiones: dónde y cómo se consigue (comerciando, mediante conquistas) y si su transporte requiere algún medio o técnica especial. Si no es accesible, ¿de dónde se trajo? ¿Existían vías comer- 
ciales? ¿Cómo se pagó? ¿Se consiguió gracias a la conquista de algún lugar? ¿Dónde pudo ser?

3. ¿Qué grado de elaboración implica el material de que está hecho? Los distintos materiales requieren mayor o menor elaboración. Una punta de flecha o el David de Miguel Ángel requieren ser cortados y tallados; el metal de una espada requiere conocimientos sobre extracción, fundición y aleación. ¿Por qué es importante que el alumno considere el grado y tipo de elaboración del material? La elaboración requiere saber cómo hacer algo, lo que tiene que ver con el grado de desarrollo del conocimiento tecnológico, y según el tipo de elaboración puede requerirse más o menos mano de obra especializada y liberada de otros trabajos. Así, esta pregunta debe llevar al planteamiento de las restantes cuestiones.

4. ¿Qué tipo de conocimientos tecnológicos implica la elaboración del objeto? Construir el Partenón o un canal con esclusas requiere, además de conocimientos sobre cómo trabajar los materiales, conocimientos de diferente complejidad sobre diseño, ensamblaje, fuerzas y estructuras, lo que también tiene que ver con el desarrollo de los conocimientos tecnológicos y puede demandar distinta cantidad de mano de obra especializada.

5. ¿Qué cantidad de personas se requieren para la elaboración del objeto analizado? La elaboración de un objeto supone concebirlo, conseguir los materiales, transformarlos y ensamblarlos. ¿Por qué es importante que el alumno se pregunte por el número de personas necesarias para realizarlo? Si la elaboración requiere muchos trabajadores, puede implicar una organización más o menos compleja de los mismos, y si se requieren trabajadores liberados de tener que conseguir su sustento por otras vías, debe haber alimentos en excedente para su mantenimiento.

6. ¿Qué grado (y tipo) de organización social y política parece necesaria para la elaboración del objeto? Una sola persona podría tallar con una piedra la punta de una flecha, pero la construcción de una catedral requiere un elevado grado de organización del trabajo y personas con conocimientos y autoridad para dirigir a otras. A mayor número de personas trabajando en algo, mayor probabilidad de que surjan conflictos por las posibles situaciones injustas derivadas de la distribución del trabajo, las diferencias en las condiciones laborales y las diferencias en el pago. Consecuentemente, si el objeto sugiere una organización social 
compleja, implica que ha sido necesario algún tipo de norma para resolver los conflictos y de poder efectivo capaz de establecer el orden.

7. ¿Qué recursos económicos se requieren para la realización del objeto analizado? Los recursos económicos necesarios para construir una punta de flecha, un trillo o un acueducto son diferentes. Además del tipo de materiales, la complejidad y la envergadura del objeto, afectan a su costo el número de trabajadores, lo que se les pague o dé a cambio, el tipo de tecnología (que puede atenuar o incrementar los trabajadores necesarios), los 'impuestos' que recaudan aquellos que ejercen la autoridad y establecen el orden... Si los recursos necesarios son muchos, ¿de dónde han salido?

8. ¿Es necesario algún tipo de condición especial (política, económica u organizativa) para que se den los recursos económicos necesarios? Es necesaria la existencia de un poder u organización que permitan recaudar los excedentes de recursos (procedan o no de la riqueza natural de la zona) y asignarlos a la producción del objeto en cuestión. Pero, ¿qué tipo de condición (poder u organización) puede posibilitar la financiación de la construcción del objeto? Puede ser el poder coercitivo, como en el caso de las pirámides de Egipto, o puede ser la inversión económica voluntaria de particulares a través de una organización financiera más o menos desarrollada, como ocurrió en Inglaterra con la construcción de canales durante la Revolución Industrial.

9. ¿Qué tiempo se requiere para la realización del objeto en cuestión? ¿Y para su difusión? La naturaleza del objeto (materiales y su disponibilidad, complejidad del mismo) y de las condiciones demográficas, técnicas, económicas, políticas y financieras necesarias para su producción sugieren un tiempo necesario para la realización del objeto. Igualmente, para que el objeto construido tenga consecuencias visibles se requiere también tiempo, aunque este impacto puede variar dependiendo de distintos factores.

10. ¿Qué valores, creencias, metas, sensibilidad y personalidad refleja la existencia, naturaleza y (eventualmente) el contenido del objeto? Unas determinadas condiciones (naturales, demográficas, técnicas...) son necesarias para que puedan construirse los objetos, pero existe además una intención que obedece a los valores, creencias, metas, sensibilidad y personalidad de quienes deciden construir el objeto o apoyan la idea. Por ejemplo, la inversión de 
recursos en monumentos funerarios como las pirámides sugiere la importancia que los constructores daban a la vida después de la muerte. Existe una interacción recíproca entre las condiciones y los valores que subyace al cambio socio-histórico y que resalta la necesidad de que el alumno se pregunte por el papel desempeñado por estos.

\section{1. ¿Qué impacto ha podido tener el objeto analizado (o el conjunto de los existentes)} en la vida y cambio social? Además de enseñar al alumnado a preguntarse por los factores que explican la existencia del objeto analizado, es importante enseñarles a preguntarse por las consecuencias probables de su existencia. Esta puede explicar cambios posteriores, como su posible repercusión en aspectos concretos de la vida o en los cambios demográficos, tecnológicos, sociales, militares, políticos y culturales. Además, los objetos (herramientas, armas, templos, cuadros...) pueden agruparse en categorías con similar repercusión en la vida social, lo que permite inferir los efectos que tendría hoy la creación de un objeto análogo.

Hasta aquí se ha comentado el conjunto de preguntas que como mínimo los alumnos deberían ser capaces de plantearse frente a cualquier DNE. Esto no implica que necesariamente tengan que preguntarse siempre por todas ellas, aunque sí hace falta que sean capaces de hacerlo. Pero, ¿ser capaz de ello es realmente un indicador de competencia? Ciertamente. Adquirir la capacidad de analizar y comprender el significado histórico-social de un DNE supone adquirir la capacidad de formularse preguntas que impliquen la capacidad de valorar el documento desde diferentes perspectivas.

Ser capaces de deducir el significado del objeto en relación con cada dimensión de análisis. Por otra parte, comprender el significado histórico-social de un DNE supone ser capaz de formular respuestas hipotéticas a las preguntas planteadas, lo que dependerá de los conocimientos previos sobre los pueblos, su organización, su cultura y los procesos de cambio. Sin embargo, las respuestas sólo serán indicativas de capacidad de comprensión y aplicación de conocimientos cuando tengan que aplicarlos a elementos no vistos previamente. De no ser así, su respuesta podría ser meramente el resultado de memorizar sin comprender. Por ejemplo, si se presenta una fotografía del acueducto de Segovia, es muy probable que lo reconozcan y hablen sobre las obras civiles de los romanos simplemente porque lo han memorizado pero, si en lugar de este acueducto se muestran los restos de otro acueducto desconocido 
para ellos, el hecho de que sean capaces de deducir las características probables del pueblo que lo construyó sí constituiría un indicador de comprensión.

\section{Principios para la Evaluación la Comprensión del Significado de DNE}

Las consideraciones descritas sugieren que los alumnos deben conseguir varios objetivos relativos a la comprensión de los DNE, lo que exige considerarlos todos al plantear la evaluación. Esta debe incluir los siguientes elementos.

Primer criterio: Pedir la descripción de la fuente. Ya que es necesario identificar el objeto para poder extraer más información sobre su contexto, esta pregunta debería plantearse siempre, salvo que se trate de un objeto muy familiar.

Segundo criterio: Evaluación del esquema de análisis alumno. La adquisición del esquema de análisis constituye un objetivo de aprendizaje, por lo que es necesario evaluar expresamente si se ha interiorizado el esquema de preguntas. Para esto, puede pedirse al alumno que indique qué aspectos del objeto analizaría (no que lo analice) para obtener información sobre su contexto. Para evitar que los alumnos memoricen las preguntas sin interiorizar el esquema de análisis, se puede evitar presentar durante la enseñanza la lista de cuestiones pero usarlas cada vez que aparezca un DNE, de modo que el alumno pueda ir asimilando el procedimiento. También puede pedirse un análisis completo y sistemático de la información que proporciona el objeto sobre la sociedad que lo creó y deducir a partir del mismo el esquema de análisis en que se han basado.

Tercer criterio: Evaluación de la capacidad de analizar la fuente documental desde distintas perspectivas diferentes de la habitual. Es importante que los alumnos sean capaces de analizar la fuente no sólo desde la perspectiva más habitual (religiosa, artística...), sino también desde otras perspectivas (demográfica, económica, técnica, política, cultural...). Por ello, es importante plantearles directamente cuestiones que pongan de manifiesto si son capaces de responderlas. Un ejemplo sería: (Dado un dibujo de un templo griego no familiar) Describe qué condiciones demográficas (o económicas, políticas, tecnológicas, etc.) probablemente hicieron posible un templo como el del dibujo. Justifica tu respuesta. 
Cuarto criterio: Evaluación de la capacidad de aplicar al análisis de DNE modelos conceptuales predeterminados. Los DNE pueden servir también como base para evaluar la capacidad de utilizar los esquemas de representación de la realidad social de un momento dado o de alguno de sus aspectos. Por ejemplo, si se han trabajado las semejanzas y diferencias entre el Paleolítico y el Neolítico, se puede preguntar, dado un arpón de hueso, con cuál de las dos culturas se relaciona y por qué. En este caso, sin embargo, no se evalúa tanto la capacidad de extraer información de la fuente sino más bien la calidad de los conocimientos que tienen que servir como base para valorar el objeto analizado.

Quinto criterio: Evaluación de la capacidad de ubicar el objeto en el espacio y en el tiempo. Por ejemplo: (Dado el dibujo de un paisaje en el que aparecen fábricas primitivas) ¿A qué lugar y tiempo es probable que pertenezca el paisaje? Es habitual pedir a los alumnos la ubicación espacio-temporal como paso previo para poder comprender el valor informativo, pero en ese caso se espera que el alumno recuerde algo aprendido, con lo que se evalúa conocimiento factual. Desde el marco de evaluación propuesto, el proceso debe ser el inverso: llegar a la ubicación como consecuencia del análisis. Para evaluar la comprensión del documento, es importante preguntar en términos de probabilidad y pedir que el alumno lo deduzca del análisis realizado.

\section{Objetivos e hipótesis}

Dado que no hay estudios previos sobre el tema, este trabajo pretende responder a una pregunta: El modo en que los profesores evalúan la capacidad de comprender documentos no escritos, ¿responde al modelo teórico expuesto u obedece a otra concepción del significado de esta competencia? Además, en base a las evidencias ya discutidas, esperamos que los profesores tiendan a utilizar tareas de baja complejidad, principalmente de conocimiento factual.

\section{Método}

\section{Participantes}

Un total de 148 profesores de Primaria que realizaban estudios de Psicopedagogía y tenían un mínimo de tres meses de experiencia profesional participaron en el estudio voluntariamente. Fueron escogidos por estar preparándose para ser profesores de Educación Secundaria y Orientadores escolares, nivel educativo en el que se debe trabajar y evaluar la comprensión de DNE. El 84.5\% eran mujeres y la edad media se situó entre los 23 y 26 años. 


\section{Materiales}

Se presentó a los profesores la tarea que aparecen en la Figura 2.

A continuación se enumeran una serie de documentos no escritos. Suponiendo que se presentaran a los alumnos, ¿qué preguntas seria necesario plantearles si quisiéramos evaluar si comprenden su valor informativo en relación con la sociedad que los creó y, en este caso, a qué se debe? Escoge dos objetos para responder.

1. Una punta de flecha de piedra

2. Una espada de hierro

3. El Partenón

4. Una moneda

5. Una herradura

6. Un trirreme griego

7. Una noria

8. La pirámide de Chichen Itza

9. Un canal inglés con esclusas

10. El grabado de la derecha

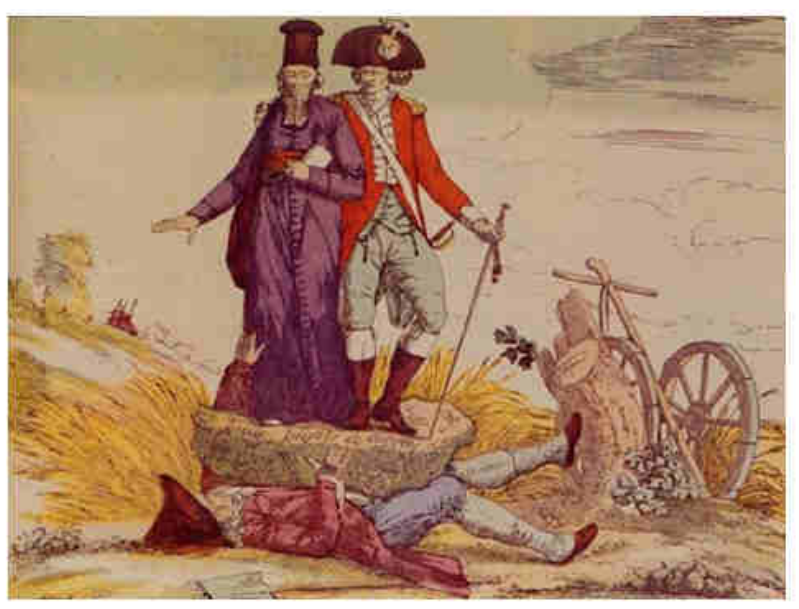

Figura 2. Tarea presentada para valorar la forma de evaluar la comprensión de DNE.

\section{Procedimiento}

Se presentó la tarea a los profesores y se les preguntó si entendían las instrucciones. Se resolvió cualquier duda que surgiera y se les dejó el tiempo necesario para completarla. Las tareas que diseñaron los profesores se codificaron según cinco categorías definidas en base al modelo propuesto de comprensión de DNE, a las cuales se añadieron dos más para incluir las preguntas no clasificables en las demás. Dos expertos en la enseñanza de Historia en Secundaria avalaron la adecuación tanto de las categorías propuestas como de los criterios de clasificación diseñados (recogidos en la Tabla 1 junto con ejemplos de preguntas). Este hecho constituye un indicador de la validez de contenido del instrumento. Los autores codificaron 
las preguntas independientemente, llegando a un acuerdo cercano al 100\%. Cuando hubo discrepancias, se resolvieron mediante un análisis conjunto del caso.

\section{Análisis Estadístico}

Se calculó el porcentaje de profesores que había planteado preguntas pertenecientes a las distintas categorías para explorar diferencias en el grado en que se plantean los diferentes tipos de preguntas y su ajuste al modelo teórico presentado. Para comprobar la posible existencia de 'estilos' de evaluación definidos por el uso sistemático de preguntas de diferentes categorías, se realizó un análisis loglineal jerárquico, adecuado para detectar asociaciones entre variables categóricas (Pardo y Ruiz, 2012).

\section{Resultados}

Ajuste del Modo de Evaluación de DNE de los Profesores al Modelo Teórico

La Tabla 1 muestra el porcentaje de profesores que ha planteado preguntas referentes a cada categoría.

Tabla 1. Categorías de clasificación de las preguntas para evaluar la comprensión del valor informativo de DNE, ejemplos de cada categoría y porcentaje de profesores que las utilizan.

C1. Petición de la descripción de la fuente. Criterios de codificación: Pedir la descripción de las características, la identificación de la naturaleza y de la función probable. Ejemplo: ¿Cuál crees que podría ser la función probable de este objeto?

C2. Evaluación del esquema de análisis alumno. Criterios de codificación: Pedir que indiquen qué aspectos del objeto analizarían para obtener la información que contiene sobre el contexto. Ejemplo: ¿Qué preguntas sobre el objeto te harías para obtener información sobre el contexto en que se generó?

C3. Evaluación de la capacidad de analizar la fuente documental desde distintas perspectivas, diferentes de la habitual. Criterios de codificación: Pedir la formulación de hipótesis. Ejemplo: ¿Qué podría decirnos el grado de elaboración objeto sobre los conocimientos tecnológicos del contexto en que se creó?

C4. Evaluación de la capacidad para identificar información necesaria para el con- 
traste de hipótesis. Criterios de codificación: Preguntar qué información es necesario buscar para contrastar las hipótesis. Ejemplo: ¿Qué información necesitarías obtener para ver si tu hipótesis es cierta?

C5. Evaluación de la capacidad de ubicar el objeto en el espacio y en el tiempo. Criterios de codificación: Pedir la deducción del contexto espacio-temporal a partir de las características del objeto. Ejemplo: En base a las hipótesis que has generado sobre el contexto en el que surgió este objeto, ¿en qué momento de la historia lo ubicarías de forma probable? ¿Por qué?

C6. Preguntas ambiguas. Criterios de codificación: Pedir la información de manera muy general. Ejemplo: ¿Qué te dice este objeto?

C7. Conocimiento de hechos. Evaluación no relevante para el propósito que se persigue. Criterios de codificación: Realizar preguntas que no impliquen la deducción de información a partir del objeto. Ejemplo: ¿Cómo se fabrica este objeto? ¿Dónde está situado? ¿A qué época pertenece?

Como puede observarse, existen diferencias muy grandes en el grado en que los profesores utilizan cada categoría de preguntas (del 2.7\% al 96.6\%). A menudo, los profesores incluyen únicamente preguntas de la categoría de descripción de la fuente (58.8\%). El 23.0\% solicita la formulación de hipótesis en sus tareas de evaluación, el 8.1\% trata de evaluar el esquema de análisis del alumno, el 2.7\% su capacidad para identificar información necesaria para contrastar hipótesis y el $7.4 \%$ su capacidad para deducir en base al análisis realizado la ubicación espacio-temporal. Por otro lado, el 29.1\% de los profesores incluye preguntas excesivamente ambiguas y el $96.6 \%$ plantea preguntas sobre conocimiento de hechos.

\section{Perfiles de Evaluación de la Comprensión de DNE}

La Tabla 2 presenta el modelo resultante del análisis loglineal realizado sobre la posible existencia de asociaciones entre el planteamiento de distintos tipos de preguntas.

Tabla 2. Modelo resultante del análisis log-lineal jerárquico.

\begin{tabular}{|c|c|c|c|c|c|}
\hline$\overline{\text { Paso }}$ & & Efectos $^{1}$ & Chi-cuadrado & $\mathrm{gl}$ & Sig. \\
\hline 102 & Clase generadora & $\begin{array}{c}1 * 5,2 * 4,2 * 5,1 * 3 * 6,1 * 3 * 7 \\
2 * 3 * 6,3 * 4 * 6,4 * 6 * 7\end{array}$ & 19.776 & 101 & 1.000 \\
\hline
\end{tabular}


Nota. ${ }^{1}$ Los números de la columna Efectos hacen referencia a las categorías numeradas de la Tabla 1.

El análisis proporcionó un modelo con excelente ajuste (razón de verosimilitudes: $\chi^{2}=$ $19.776, p=1.000$ ). La diferencia máxima entre valores observados y valores marginales ajustados fue de .208, por debajo del criterio de convergencia (.250). Todos los parámetros mostrados resultaron tener efectos no nulos $(p<.05)$ en el paso anterior $\mathbf{y}$, por tratarse de un modelo jerárquico, incluyen los efectos de orden inferior (interacciones dobles y efectos principales).

Los efectos encontrados fueron interpretados mediante los residuos tipificados de las tablas de contingencias (Pardo y Ruiz, 2012), que cuando son superiores a una desviación típica (mayor de 1.96 o menor de -1.96) indican que existe una asociación. A continuación se describen las tendencias encontradas, indicando entre paréntesis el valor absoluto de los residuos tipificados correspondientes para facilitar la interpretación de su magnitud.

a) Los profesores que piden al alumno la descripción tienden a pedirle también la deducción -no recuerdo- de la ubicación espacio-temporal (C1/C5) (|2.2|).

b) Cuando se pregunta qué aspectos del objeto se analizarían para obtener información sobre su contexto y por qué, se tiende a preguntar qué información se necesitaría para contrastar las hipótesis (C2/C4) (|5.0|), pese a no haberse pedido formularlas.

c) La evaluación del esquema de análisis también tiende a asociarse a la de la deducción de la ubicación espacio-temporal (C2/C5) (|4.7|). Tanto este esquema como los anteriores muestran interés por evaluar la comprensión y la capacidad de razonar.

d) Cuando se pide formular hipótesis, suelen realizarse preguntas ambiguas. Esta asociación tiende a ocurrir cuando no se pide la descripción de la fuente. (C1/C3/C6) (|3.4|). Por ejemplo, no pedir la descripción (contexto ambiguo) y preguntar ‘¿Qué te dice este objeto sobre la sociedad que lo ha utilizado?' (pregunta ambigua) y ‘¿Qué recursos tecnológicos pueden haber sido necesarios para elaborarlo?' (petición de formulación de hipótesis). Este esquema muestra confusión en el profesor sobre la evaluación de la competencia.

e) En los casos en que se pide descripción de la fuente y formulación de hipótesis, tiende a no preguntarse por conocimiento factual (C1/C3/C7) $(|2.7|)$. Cuando se pide descrip- 
ción pero no formulación de hipótesis, sí suele preguntarse por conocimiento de hechos, caso en que el profesor no evalúa comprensión, sino recuerdo.

f) Solicitar la formulación de hipótesis suele asociarse a la realización de preguntas ambiguas, pero solo cuando no se evalúa el esquema de análisis del alumno. (C2/C3/C6) $(|2.5|)$.

g) Cuando se realizan preguntas ambiguas y se pide también la formulación de hipótesis, se tiende a pedir la identificación de la información necesaria para contrastar éstas (C3/C4/C6) (|2.1|), esquema que muestra interés por evaluar la comprensión.

h) Por último, si se pide identificar información necesaria para contrastar hipótesis, existe una fuerte tendencia a no realizar preguntas sobre conocimiento de hechos, lo que suele ocurrir cuando no se realizan preguntas ambiguas (C4/C6/C7) (|8.3|).

\section{Discusión}

Este trabajo perseguía, en primer lugar, exponer un modelo de evaluar la comprensión de DNE y, en segundo lugar, determinar si el modo en que los profesores la evalúan se ajusta a dicho modelo. Idealmente, los profesores deberían haber propuesto preguntas de cada categoría, evitando las preguntas ambiguas y las preguntas de conocimiento factual. Los resultados han puesto de manifiesto varios hechos importantes que se discuten a continuación.

En primer lugar, prácticamente la totalidad de los profesores plantean preguntas sobre conocimiento factual, que pueden tener un gran valor como indicadores de otras competencias pero no resultan adecuadas para evaluar la comprensión de DNE: el alumno podría fallar la pregunta pese a comprender el valor informativo del documento o contestar correctamente sin haberlo comprendido en realidad. Este resultado concuerda con los encontrados en el trabajo de Alonso-Tapia y Hernansaiz Garrido (2013) y apoya la expectativa mencionada de un uso elevado de preguntas sobre conocimiento factual. En segundo lugar, casi un tercio del profesorado plantea preguntas ambiguas que dificultan que el alumno sepa qué es lo que busca el profesor e impiden, por tanto, la evaluación adecuada de la competencia y la identificación de las dificultades del alumado.

En tercer lugar, el 40\% de profesores no solicitan la descripción de la fuente (condición necesaria pero no suficiente para comprenderla). En cuarto lugar, un escaso porcentaje de 
profesores plantea preguntas de las categorías de complejidad media y alta (C2, C3, C4 y C5), lo que está en línea con otros estudios sobre evaluación de competencias (Alonso-Tapia et al., 2007; Alonso-Tapia y Hernansaiz-Garrido, 2013; Pérez de Landazábal et al., 2012). Por último, existen determinados patrones compuestos por asociaciones entre formas de evaluar y de no evaluar. Estos patrones presentan una diversidad que indica que la falta de sistematicidad en las evaluaciones de los profesores. No obstante, algunos profesores sí presentan patrones indicativos de que, para evaluar la competencia que nos ocupa, es necesario centrarse en la comprensión y el razonamiento, aunque el esquema que utilizan no sea completo.

Los resultados presentados indican que la mayoría de profesores no evalúa adecuadamente esta competencia, ya que utilizan preguntas ambiguas o irrelevantes y no incluyen tareas de complejidad media o elevada. Estos resultados van en la línea de otros trabajos donde se afirma que los métodos de evaluación utilizados suelen ser deficientes (Black y Wiliam, 1998, Segers et al., 2003). Además, esta deficiencia en la forma evaluar lleva, nuevamente, a que no sea posible identificar las dificultades del alumado y, por tanto, no sea posible proporcionar ayudas adecuadas a tales dificultades, obstaculizando así que se produzca una evaluación para el aprendizaje (Dochy, 2005) y que se desarrolle una adecuada motivación por aprender (Alonso-Tapia y Fernández, 2008; Alonso-Tapia y Pardo, 2006).

Este estudio presenta varios puntos fuertes de carácter tanto teórico como aplicado. En primer lugar, ha presentado un modelo de lo que implica la capacidad de comprender DNE y de cómo evaluar esta competencia con el objetivo de ayudar a los alumnos a superar las dificultades que tengan. En segundo lugar, ha puesto de manifiesto que las tareas utilizadas por los profesores reflejan la idea de que lo que hay que conocer son hechos, más que saber pensar en la línea propuesta por el modelo. En tercer lugar, existe una cierta convergencia de los resultados obtenidos con los encontrados en relación a otras competencias que contribuye a la comprensión de los supuestos desde los que los profesores evalúan. En cuarto lugar, los hechos descritos en los puntos anteriores tienen una importante implicación de carácter aplicado, a saber, la necesidad de formar al profesorado en relación a la evaluación de competencias en general y de la comprensión de DNE en particular. Concretamente, resulta necesario hacerles conscientes de la discrepancia existente entre la información que proporcionan los métodos que utilizan y el tipo de evidencia que en realidad resultaría informativa (Pérez- 
Landazábal y Moreno, 1998), con objeto de propiciar que modifiquen sus métodos habituales de evaluación.

Además, este estudio tiene varias limitaciones, entre las que destaca la muestra, la cual es relativamente pequeña, está centrada en profesores de Educación Primaria (aunque preparándose para trabajar en Secundaria como orientadores) y mayoritariamente compuesta por mujeres. Esto hace necesario que posteriores investigaciones repliquen estos resultados en muestras de mayor tamaño y diversidad de género, y en diferentes niveles educativos. Además, el tipo de diseño y la naturaleza de los datos ha impedido responder preguntas importantes; por ejemplo, en qué medida el profesorado se beneficiaría de recibir información específica sobre el modo de enseñar y evaluar la comprensión de documentos no escritos. Futuros estudios que partan de esta base se beneficiarían de recoger datos diferentes (e.g., diseños prepost) y realizar análisis diferentes a los utilizados en el presente estudio.

\section{Referencias}

Alonso-Tapia, J. (1992). Leer, comprender y pensar: Desarrollo de estrategias y técnicas de evaluación. Madrid, España: Ministerio de Educación y Ciencia - CIDE.

Alonso-Tapia, J. (1997). Evaluación del conocimiento y su adquisición. Madrid, España: Ministerio de Educación y Ciencia - CIDE.

Alonso-Tapia, J. (2001). La evaluación de la competencia curricular en el contexto de la orientación educativa. Revista de Española de Orientación y Psicopedagogía, 12(21), 1538. Obtenido de http://www.uned.es/reop/pdfs/2001/12-21\%20-\%20Jesus\%20Alonso.pdf

Alonso-Tapia, J., Asensio, F., López, I., Carriedo, N., y Rychecki, F. (2007). Assessment for Learning: Social Science teachers' ideas on assessment of causal understanding. Electronic Journal of Research in Educational Psychology, 5(13), 593-616. Obtenido de http://www.investigacion-

\section{psicopedagogica.org/revista/new/english/ContadorArticulo.php?190}

Alonso-Tapia, J., y Fernández, B. (2008). Development and initial validation of the classroom motivational climate questionnaire (CMCQ). Psicothema, 20(4), 883-889. Obtenido de http://www.psicothema.com/psicothema.asp?id=3570

Alonso Tapia, J., y Hernansaiz Garrido, H. (2013). Evaluar para el aprendizaje. Problemas en la práctica de la evaluación de competencias: el caso de la comprensión de tablas y gráfi- 
cos. Revista Educativa Hekademos, 13, 7-18. Obtenido de http://hekademos.com/hekademos/content/view/281/32/

Alonso-Tapia, J., y Pardo, A. (2006). Assessment of learning environment motivational quality from the point of view of secondary and high school learners. Learning and Instruction, 16(4), 1-15. DOI: 10.1016/j.learninstruc.2006.07.002

Black, P., y Wiliam, D. (1998). Assessment and classroom learning. Assessment in Education: Principles, Policy \& Practice, 5(1), 7-74. DOI: 10.1080/0969595980050102

Dochy, G. (2005). 'Learning lasting for life' and 'assessment': How far did we progress? Presidential address, EARLI-2005. Nicosia, Cyprus.

Mingorance Arnáiz, C., y Calvo Bernardino, A. (2013). Los resultados de los estudiantes en un proceso de evaluación con metodologías distintas. Revista de Investigación Educativa, 31(1), 275-293. DOI: 10.6018/rie.31.1.153291

Oldroyd, D. (1999). Non-written Sources in the Study of the History of Geology: Pros and Cons, in the Light of the Views of Collingwood and Foucault. Annals of Science, 56(4), 395-415. DOI: $10.1080 / 000337999296355$

Pardo, A., y Ruiz, M. A. (2012). Análisis de datos en ciencias sociales y de la salud (vol. III). Madrid, España: Síntesis.

Pérez de Landazábal, Mª C., y Moreno, J. M. (1998). Evaluación y detección de dificultades en el aprendizaje de física y química en el segundo ciclo de la ESO. Madrid, España: CIDE-MEC.

Pérez de Landazábal, C., Varela, P., y Alonso-Tapia, J. (2012). Assessment for learning: Science teachers' ideas on assessment of core competences in Science understanding. Journal for the Study of Education and Development, 35(2), 215-232. DOI: $\underline{10.1174 / 021037012800218023}$

Segers M., Dochy F., y Cascallar E. (2003). Optimising new modes of assessment: in search of qualities and standards. Dordrecht/Boston/London: Kluwer.

Trepat i Carbonell, C. A. (1995): Procedimientos en Historia. Un punto de vista didáctico. Barcelona, España: Graó/ICE de la Universidad de Barcelona.

Waite, G. (1987). Public health in pre-colonial east-central Africa. Social Science \& Medicine, 24(3), 197-208. DOI: 10.1016/0277-9536(87)90047-5 